\title{
Estudo das propriedades do Diodo Emissor de Luz (LED) para a determinação da constante de Planck numa maquete automatizada com o auxílio da plataforma Arduíno
}

\author{
Study of properties of Light Emission Diode (LED) for the determination of the Planck constant in a \\ automated device with Arduino platform aid \\ Ivanor N. de Oliveira ${ }^{1}$, Jorge A. P. Ramos*10, Wilton L. Silva ${ }^{2}$, Valteni D. Chaves ${ }^{3}$, \\ Clênia A. O. de Melo ${ }^{1}$
${ }^{1}$ Universidade Estadual do Sudoeste da Bahia, Ciências Exatas e Tecnológicas, Vitória da Conquista, BA, Brasil
${ }^{2}$ Instituto Federal de Educação Ciência e Tecnologia da Bahia, Salvador, BA, Brasil
${ }^{3}$ Instituto Federal Baiano, Salvador, BA, Brasil

Recebido em 05 de Maio de 2019. Revisado em 17 de Junho de 2019. Aceito em 28 de Julho de 2019.

\begin{abstract}
No presente artigo, apresenta-se o método experimental de determinação da constante de Planck, uma das constantes físicas fundamentais, através do estudo das propriedades quânticas e ondulatórias do diodo emissor de luz (LED) numa maquete experimental automatizada, que contém um circuito eletrônico ligado a um computador utilizando o protocolo USB com o auxílio da plataforma Arduino. Os LEDs têm encontrado diversas aplicações, por exemplo: na iluminação doméstica, pública, nos semáforos, nos monitores de TV, computadores, para ler discos de CD e DVD em unidades de computador e em unidades de console de jogos, ou para transmitir sinais de controles remotos para equipamentos eletrônicos. O LED é uma ligação vital entre eletrônica e fotônica os lasers de semicondutor baseados em LEDs enviam sinais ópticos modulados através de fibras ópticas, atendendo à demanda cada vez maior da telecomunicação de banda larga e Internet. A automatização das medições e da elaboração dos dados dos experimentos de Física permite aos alunos aprofundar o conhecimento de métodos e técnicas do experimento, estudar aparelhos de pesquisa científica, dominar diferentes métodos de pesquisas experimentais, dominar métodos matemáticos e gráficos dos resultados obtidos e também a avaliação das medições realizadas.

Palavras-chave: Ensino de Física. Laboratório remoto. Plataforma Arduíno.
\end{abstract}

In the present article, the experimental method of determination of Planck's constant, one of the fundamental physical constants, is presented by studying the quantum and wave properties of the light emitting diode (LED), in an automated experimental model which contains an electronic circuit connected to a computer using the USB protocol with the help of the Arduino platform. LEDs have found a variety of applications, for example: in home, public, in-house lighting, TV monitors, computers, to read CD and DVD discs in computer units and game console units, or to transmit signals from remote controls to electronic equipment. LED is a vital link between electronics and photonics: LED-based semiconductor lasers send modulated optical signals through fiber optics, catering to the ever-increasing demand for broadband and Internet telecommunication. The automation of measurements and data processing of Physics experiments allows students to deepen their knowledge of methods and techniques of the experiment, to study scientific research apparatus, to master different experimental research methods, to master mathematical methods and graphs of the results obtained, and also the evaluation of the measurements made.

Keywords: Physics Teaching. Remote laboratory. Arduino Platform.

\section{Introdução}

A descoberta do diodo emissor de luz (LED) está relacionada a dois nomes: Henry Joseph Round no Laboratório de Marconi observou o fenômeno de eletroluminescência como um fenômeno em 1907 usando um cristal de carboneto de silício e um detector [1], [2], independentemente, o cientista Russo, Oleg Vladimirovitch Losev, descobriu o LED [3] Em seus artigos científicos, publicados entre

*Endereço de correspondência: jorge@uesb.edu.br.
1924 e 1930, Oleg V. Losev forneceu um estudo abrangente sobre o LED e delineou as suas aplicações. As suas pesquisas foram publicadas em revistas científicas da União Soviética, Alemanha e Inglaterra, entretanto elas não foram postas em prática durante várias décadas [4], [5]. Ele entendeu a natureza "fria"(não térmica) da emissão do LED, mediu seu atual limiar de emissão de luz, reconheceu que sua emissão está relacionada à ação de diodo e mediu, detalhadamente, a dependência 
tensão-corrente característica desse dispositivo eletrônico, mostrada na Figura 1$][3]$.

Chama a atenção o fato de que Oleg Losev, um jovem técnico sem titulações científicas, conhecia os desenvolvimentos contemporâneos em Física [5]. Ele usou a teoria quântica de Einstein para explicar a ação do LED e chamou o processo de emissão de "efeito fotoelétrico inverso". Além disso, ele propôs uma fórmula [6] relativa à queda de tensão no contato do diodo, $\mathrm{U}$, a carga do elétron $e$, e a frequência de emissão da luz, $\nu$, através da constante de Planck, $h$ :

$$
\nu=\frac{e U}{h}
$$

A fórmula proposta por Oleg Losev, em 1929, resulta da igualdade entre as expressões quantitativas clássica e quântica da energia:

$$
e U_{o}=h \nu
$$

onde $U_{o}$ é a tensão limiar de emissão do LED.

No presente artigo, apresenta-se o método experimental para determinação da constante de Planck, uma das constantes físicas fundamentais, através do estudo das propriedades quânticas e ondulatórias do diodo emissor de luz (LED), numa maquete experimental automatizada que contém um circuito eletrônico ligado a um computador utilizando o protocolo USB com o auxílio da plataforma Arduíno. Esse componente eletrônico desempenha um papel importante na tecnologia atual. As suas aplicações estão presentes em diversas esferas da vida cotidiana, no trabalho, na vida doméstica e em ambientes públicos. Para exemplificar, vamos citar apenas algumas das suas aplicações. Ele é usado na iluminação doméstica, nos semáforos, na iluminação pública, nos monitores de TV, computadores, para ler discos de CD e DVD em unidades de computador e em unidades de console de jogos, ou para transmitir sinais de controles remotos para equipamentos eletrônicos. O mais importante, no entanto, é que o LED é uma ligação vital entre eletrônica e fotônica [5]: os lasers de semicondutor baseados em LEDs enviam sinais ópticos modulados através

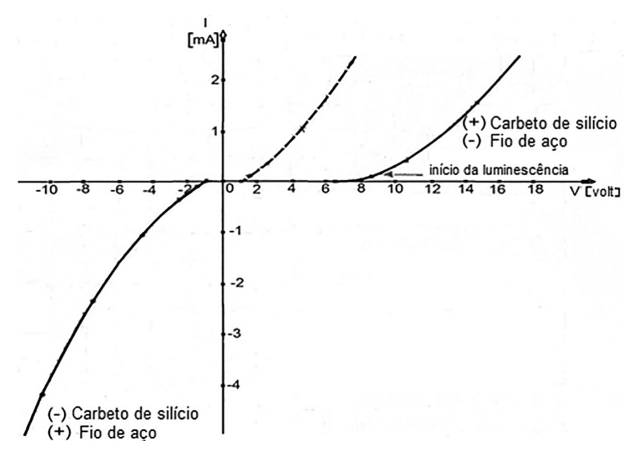

Figura 1: Gráfico das características I $\times$ V de um detector de carbeto de silício indicando limiar da emissão de luz [3]. de fibras ópticas, atendendo à demanda cada vez maior da telecomunicação de banda larga e Internet.

A automatização das medições e da elaboração dos dados dos experimentos de Física tem como objetivo, em primeiro lugar, garantir aos estudantes do Curso de Licenciatura em Física da UESB, modalidade a distância, o acesso aos laboratórios remotos de Física Geral via Internet, mas também a sua utilização pelos estudantes do curso presencial de Física, o que permite aos alunos complementar as aulas teóricas, aprofundar o conhecimento de métodos e técnicas do experimento, estudar aparelhos de pesquisa científica e a familiarização com os mesmos, dominar diferentes métodos de pesquisas experimentais, dominar métodos matemáticos e gráficos dos resultados obtidos e também a avaliação das medições realizadas.

\section{O desenvolvimento dos primeiros LEDs coloridos}

O primeiro LED de espectro visível (vermelho) foi desenvolvido em 1962 por Nick Holonyak Jr., enquanto trabalhava na General Electric. Holonyak relatou, pela primeira vez, seu LED na revista Applied Physics Letters em 1 de dezembro de 1962 7], 8. M. George Craford 9] um ex-aluno de pós-graduação de Holonyak, inventou o primeiro LED amarelo e melhorou o brilho dos LEDs vermelho e vermelho-alaranjado por um fator de dez em 1972 10]. Em 1976, T. P. Pearsall criou os primeiros LEDs de alta luminosidade e alta eficiência para telecomunicações de fibra óptica, inventando novos materiais semicondutores especificamente adaptados para comprimentos de onda de transmissão de fibra óptica [11].

\subsection{O LED azul}

Para revolucionar a tecnologia da iluminação ainda faltava desenvolver o LED azul. Como se sabe, somente a tríade formada pelas cores vermelha, verde e azul pode produzir a luz branca. Entretanto, a criação do diodo emissor de luz azul permaneceu um desafio por décadas, tanto para a comunidade científica, como para a indústria eletrônica. Os trabalhos desenvolvidos pelos cientistas japoneses Isamu Akasaki, Hiroshi Amano e Shuji Nakamura culminaram com a invenção do LED azul. Nos anos 1990 os seus trabalhos possibilitaram a produção de LEDs azuis eficientes, com estruturas complexas. Em 2014 eles receberam o Prêmio Nobel de Física pelo desenvolvimento do LED azul [12].

\section{O princípio de funcionamento do LED}

O princípio de funcionamento do LED (Figura 2 consiste da transformação da energia elétrica em radiação, que pode pertencer ao espectro visível ou infravermelho da luz.

A estrutura do LED é uma transição elétron-buraco, por isso, os mecanismos de passagem da corrente através 


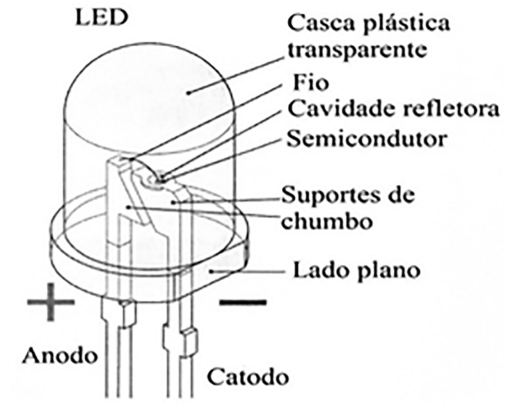

Figura 2: Partes constituintes do LED.

de um diodo de semicondutor e de um LED são iguais. Os materiais da transição elétron-buraco da estrutura do LED são escolhidos de forma que os elétrons livres na região-n (emissor) possuem menor energia que na regiãop (base). Na ausência de um campo elétrico externo, a difusão dos principais portadores de carga através da transição p-n (dos elétrons da região-n para a regiãop e dos buracos em direção contrária)é impedida pelo campo elétrico interno denominado campo de contato. A intensidade $E_{c}$ desse campo, a largura $d$ da transição p-n e a diferença de potencial de contato $U_{c}$ estão ligadas pela relação: $E_{c}=\frac{U_{c}}{d}$. Devido ao campo elétrico do contato, para a passagem de um elétron da região-n para a região-p e de um buraco em direção oposta, é necessário realizar trabalho igual ao produto da carga do elétron pela diferença de potencial de contato, ou seja, é necessário superar a barreira de potencial $e U_{c}$ (Figura 3).

Aplicando-se uma diferença de potencial $U$, diretamente polarizada, aos terminais do LED, a intensidade do campo elétrico externo E tem sentido contrário à intensidade do campo elétrico de contato $E_{c}$. Por isso, a barreira de potencial na transição p-n diminui e torna-se igual a $e\left(U_{c}-U\right)$ e parte dos portadores principais de carga tem a possibilidade de superar a transição p-n (Figura 4).

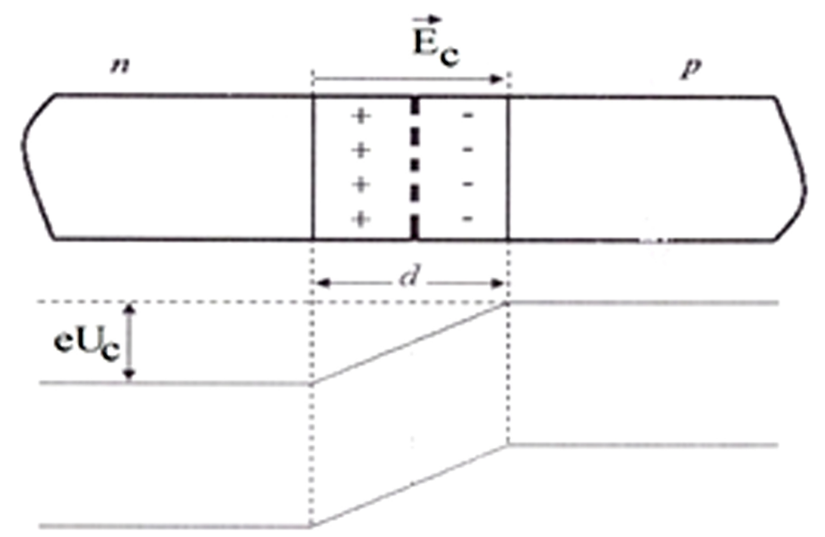

Figura 3: Campo elétrico interno $\mathrm{E}$ e barreira potencial de contato $e U_{c}$.

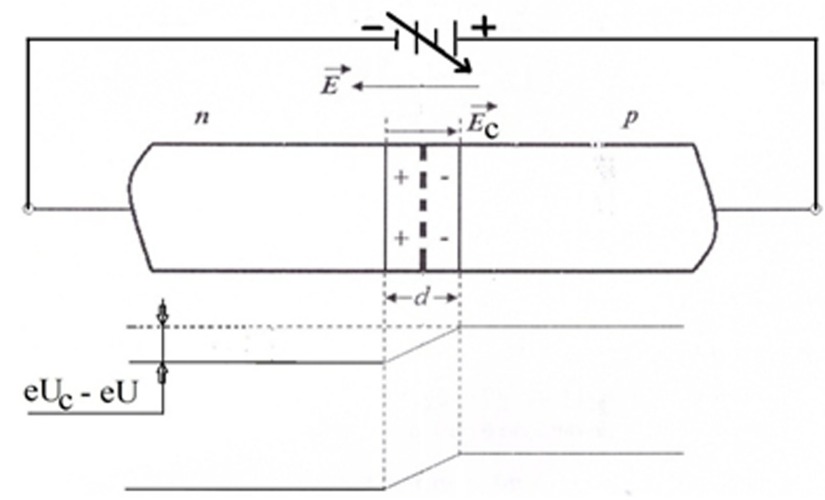

Figura 4: Campos elétricos externo $\mathrm{E}$, interno $E_{c}$ e barreira potencial na transição p-n.

Com isso, ocorre a injeção dos elétrons e buracos nas regiões p e n, correspondentemente. Quando o valor da tensão externa $U$ torna-se próximo de $U_{c}$, a barreira de potencial $e\left(U_{c}-U\right)$ e sua espessura $d$ tendem a zero. Então, os elétrons e buracos vão ao encontro uns dos outros sem impedimento e a corrente elétrica cresce abruptamente. Ela é limitada apenas pela resistência elétrica intrínseca do diodo de semicondutor, o que leva a curva $I$ vs $U$ a possuir uma contribuição aproximadamente linear, a partir do momento em que o elétron adquire quantidade de energia necessária para efetuar a transição designada por $E_{g}$ [13], 14]. No LED, a energia da fonte de corrente elétrica, gasta para superar a barreira potencial $e U_{c}$, para cada portador, transforma-se em energia luminosa. Por isso, pode-se escrever:

$$
e U_{c}=h \nu
$$

onde, a frequência da radiação do LED está ligada com o comprimento de onda, $\nu=c / \lambda$, onde $c$ é a velocidade da luz no vácuo. $\mathrm{O}$ comprimento de onda $\lambda$ pode ser medido com o auxílio de uma rede de difração.

\section{Variantes de montagem da maquete experimental automatizada para a determinação da constante de Planck}

Para a determinação da constante de Planck, são conhecidas duas variantes de montagens da maquete experimental.

\subsection{Primeira variante de montagem da maquete experimental}

A primeira variante de montagem é dada na Figura 5. constituída por uma base na qual são instalados dois suportes: um contém uma régua graduada com zero central, onde se tem um LED ligado ao circuito eletrônico da plataforma Arduíno, que se comunica com o computador via USB; o outro suporte contém uma rede de difração unidimensional de 1000 linhas por $\mathrm{mm}$. A rede de di- 


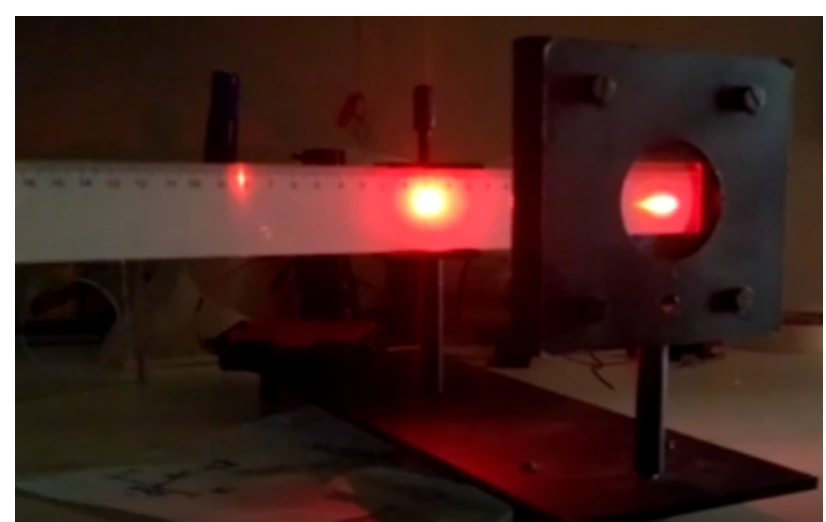

Figura 5: Maquete experimental para a determinação da constante de Planck - primeira variante de montagem.

fração unidimensional é composta por transparências paralelas para seções de luz da mesma largura, que estão localizadas no mesmo plano. As áreas transparentes são separadas por lacunas opacas. Com a ajuda dessas redes, são feitas observações na luz transmitida. O padrão de difração na rede é o resultado da interferência mútua de ondas provenientes de todas as fendas. Na Figura 5. mostra-se, com o auxílio da rede de difração, um dos máximos secundários simétricos da luz emitida pelo LED vermelho.

\subsection{Observação do espectro de difração com a primeira variante da maquete experimental}

Como mostra, esquematicamente, a Figura 6, a observação do espectro de difração da luz, nesse caso, é feita olhando-se diretamente através da rede de difração na direção da tela (ou da régua graduada) da maquete com o ambiente laboratorial escurecido. Assim é observado o espectro de difração sobre a tela ou sobre uma régua graduada com zero central, onde os máximos secundários de cada ordem se encontram em posições simétricas em relação ao máximo principal.

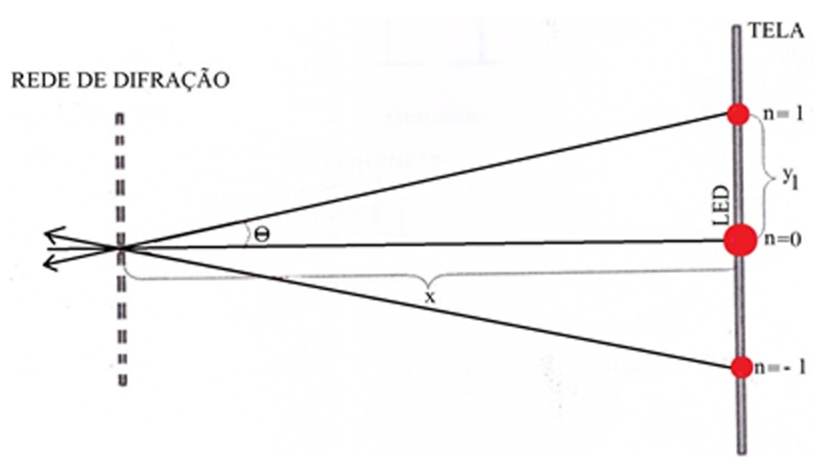

Figura 6: Esquema óptico de observação do espectro de difração da emissão do LED - primeira variante de montagem da maquete.

\subsection{Segunda variante de montagem da maquete experimental}

A segunda variante de montagem da maquete experimental permite a observação do espectro de difração da luz numa tela ou régua graduada (Figura 7) sem a necessidade de escurecer o ambiente. Ela difere da primeira pela posição do LED, agora no início da base da maquete e pela inserção de uma lente convergente antes da rede de difração. A vantagem dessa variante está na forma de observação do espectro de difração, que é visto diretamente na tela sem a necessidade de observação individual do espectro através da rede de difração (Figura 60 e em ambiente escuro.

\section{Maquete experimental construída para a determinação da constante de Planck}

A Figura 8 mostra a segunda variante de montagem da maquete experimental.

\subsection{Esquema óptico da segunda variante da maquete experimental}

O esquema óptico da segunda variante da maquete experimental, dado na Figura 9, mostra as posições de dois máximos secundários e do máximo principal do espectro de difração de um LED.

No presente trabalho nós escolhemos a segunda variante de montagem do experimento por permitir a observação do espectro de difração por todos os alunos no anteparo, com ambiente iluminado, ao passo que na primeira variante a observação do espectro é individual.

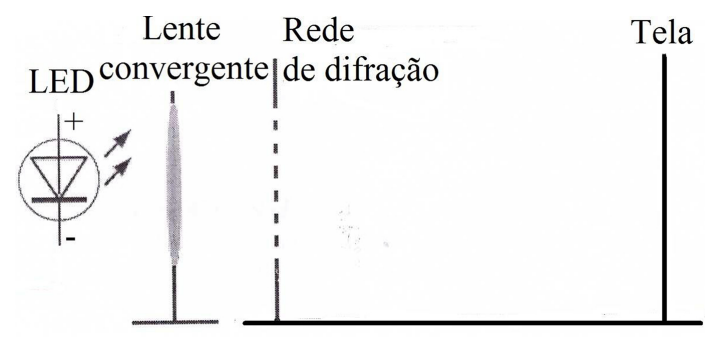

Figura 7: Esquema da segunda variante de montagem da maquete experimental.

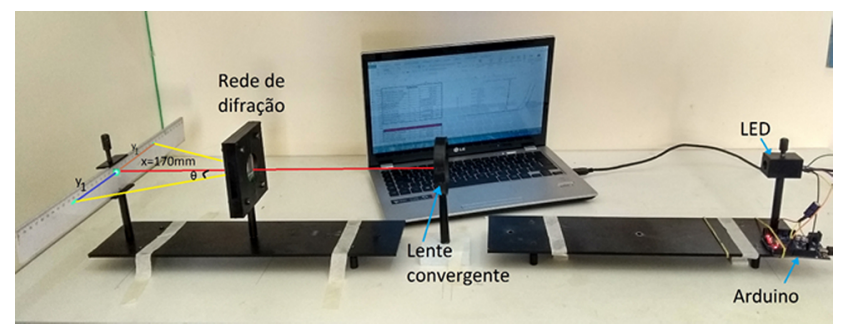

Figura 8: Segunda variante de montagem da maquete experimental para a determinação da constante de Planck. 


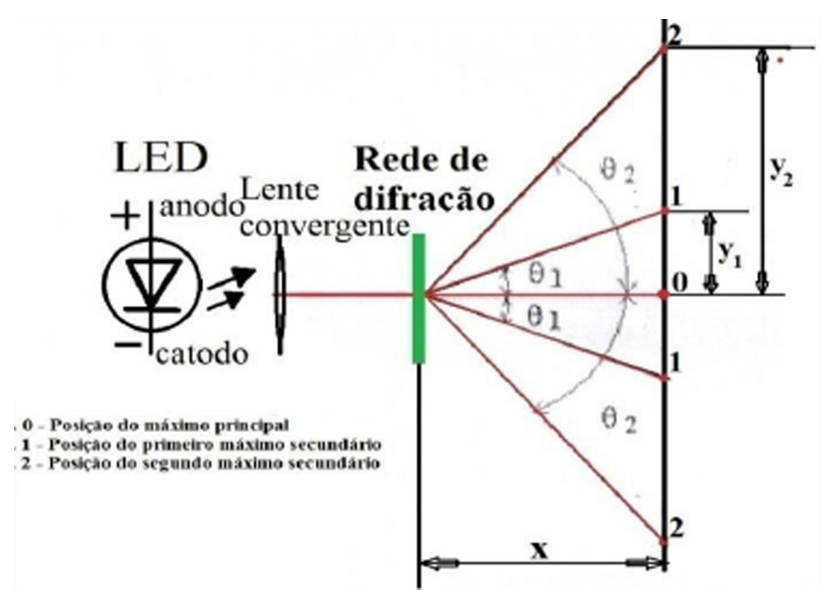

Figura 9: Esquema óptico de observação do espectro de difração da emissão do LED - segunda variante de montagem da maquete.

\subsection{Obtenção da fórmula de cálculo da constante de Planck}

De acordo com o conceito de energia como medida quantitativa das diferentes formas de movimento da matéria, podemos igualar as expressões quantitativas clássica e quântica da energia, obtidas na equação 2, e determinar o valor da constante de Planck $h$.

$$
h=\frac{e U_{o}}{\nu}
$$

A expressão da frequência linear tem a seguinte forma:

$$
\nu=\frac{c}{\lambda}
$$

onde $c$ é a velocidade da luz no vácuo.

Com o auxílio de uma rede de difração com período conhecido, pode-se determinar os comprimentos de onda da luz incidente medindo-se os ângulos de difração, segundo os quais são observados o máximo principal e um dos máximos secundários do espectro do LED. A fórmula de cálculo é a condição de formação dos máximos secundários:

$d \sin \theta=m \lambda, \quad$ assim, $\lambda=\frac{d \sin \theta_{m}}{m} m= \pm 1, \pm 2, \pm 3, \ldots$

Utilizando as equações 5 e 6 , obtemos:

$$
h=\frac{e U_{o} d \sin \theta_{m}}{m c}
$$

onde $d$ é a constante de rede.

De acordo com o esquema óptico apresentado na Figura 9 a tangente do ângulo $\theta$ é calculada pela fórmula $\tan \theta=$ $\frac{y_{m}}{x}$. O valor do ângulo $\theta$ é dado pela fórmula: $\theta=$ $\arctan \frac{y_{m}}{x}$, onde $y_{m}$ é a distância medida, através da régua, desde a ordem zero de difração (máximo central) até a ordem de difração escolhida (máximo secundário); $x$ é a distância entre a rede de difração e o ponto de luz criado na régua pela emissão do LED em ordem zero de difração.

\section{Circuito eletrônico para a medição da dependência corrente $\mathrm{x}$ tensão de um elemento eletrônico de uma maquete experimental clássica}

Inicialmente, vamos analisar o esquema do circuito de medição dado na Figura 10 A tensão de alimentação é dada em Ent.1 e Ent.2 e, como resultado, deve ser construído o gráfico da dependência da corrente através do dispositivo eletrônico (LED) versus tensão nele:

$$
I_{D} \times U_{D}
$$

ou seja,

$$
\left(U_{\text {saida } 3}-U_{\text {saida } 2}\right) \frac{1}{R_{1}} \times\left(U_{\text {saida } 1}-U_{\text {saida } 3}\right)
$$

\section{Estudo da dependência corrente/tensão do LED com o auxílio da plataforma Arduino}

O circuito da Figura 10 não pode ser ligado diretamente ao Arduíno. Ele não possui um Circuito Conversor Digital - para - Analógico (CDA). Para realizar as medições com o auxílio do Arduino, o circuito de medição foi ampliado para que, no circuito resultante, os sinais de entrada possam ser formados por PWM + LPF (Modulação da largura de pulso + RC Filtro passa-baixa) 15. A plataforma Arduino oferece uma série de vantagens em relação a outros dispositivos devido ao seu ambiente de programação simples e, claro, preços baixos e uma infinidade de placas de expansão. As placas do Arduino são construídas com base nos microprocessadores Atmel (site oficial do Atmel), bem como elementos de comunicação para programação e integração com outros circuitos. Devido às suas características técnicas e ao fato de que para o estudo da dependência corrente $\mathrm{x}$ tensão do dispositivo eletrônico nomeado acima não há exigência de velocidade, vamos utilizar a placa Arduíno Uno. Nesse sentido,

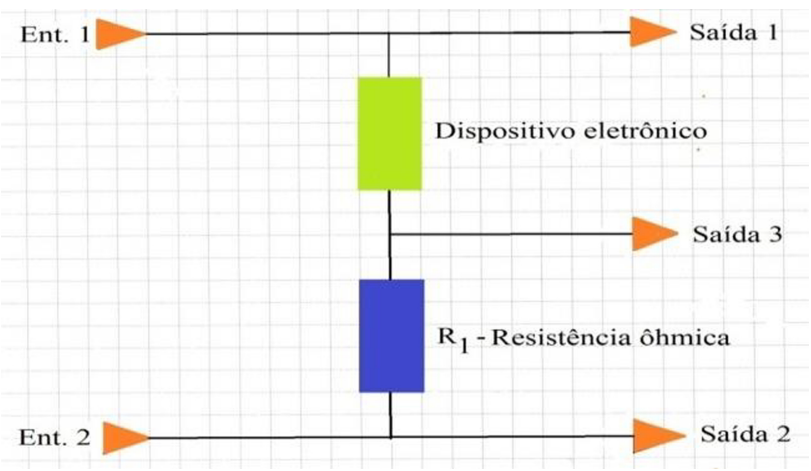

Figura 10: Esquema do circuito de medição da dependência corrente $x$ tensão. 
foi utilizado o seguinte esquema de circuito eletrônico mostrado na Figura 11

\subsection{Componentes eletrônicos utilizados}

- 1 Placa Arduíno Uno;

- 1 capacitor eletrolítico de $2200 \mu \mathrm{F}$;

- 1 resistor de $120 \mathrm{Ohm;}$

- 1 LED.

Na maquete mostrada na Figura 8 , o feixe luminoso emitido pelo LED é colimado com o auxílio de uma lente convergente e se difrata ao incidir na rede de difração. Seu espectro pode ser observado, diretamente, num anteparo (tela, régua) ou com o auxílio de uma Web-câmera instalada na maquete sem a necessidade de escurecer o ambiente.

\section{O Hardware}

Na Figura 12 é mostrada a montagem do circuito eletrônico, onde o LED encontra-se ligado em série com o resistor limitador de corrente, cuja resistência é de 120 ohm.

O terminal negativo(-) do capacitor e um dos terminais do resistor encontram-se conectados ao terminal de referência da placa Arduíno (gnd). Já o terminal positivo $(+)$ do LED e do capacitor são conectados ao pino D6 da placa Arduíno. Deve-se salientar que este pino, D6, foi configurado por software para operar como uma saída PWM (Pulse Width Modulation). Como o sinal PWM fornece uma onda de corrente/tensão pulsada, foi necessário convertê-la numa saída analógica linear. Para tanto, foi utilizado um capacitor, cuja função principal é atuar como filtro. Como se sabe, os filtros são circuitos eletrônicos que deixam passar uma determinada banda de frequência sem atenuação e suprimem todas as outras frequências. A frequência na qual a supressão começa é chamada de frequência de corte. Desta forma, é possível produzir um sinal de corrente/tensão analógico com regulação efetuada via software. Finalmente, o terminal negativo do LED é conectado ao outro terminal do resistor limitador de corrente. Para encontrar a corrente e tensão presentes no LED foi adotada a seguinte metodologia:

- A entrada analógica $A_{1}$ do Arduino, é utilizada para medir a tensão sobre o resistor, enquanto a

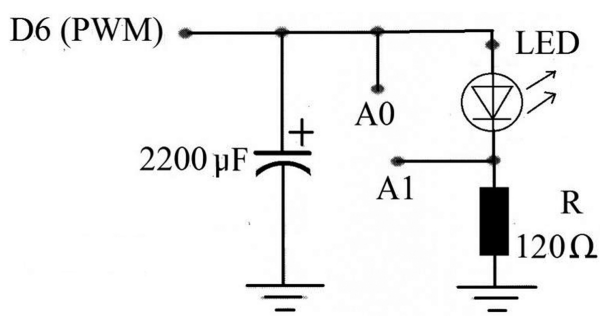

Figura 11: Esquema para medição de $\mathrm{I} \times \mathrm{V}$ [17. entrada analógica $A_{o}$ utilizada para medição da tensão total do circuito, ou seja, a tensão fornecida pela saída PWM no pino D6. Assim, é possível determinar a tensão sobre o LED subtraindo da tensão obtida na entrada $A_{o}$ aquela encontrada na entrada $A_{1}$. Logo: $V_{\text {led }}=\left(A_{o}-A_{1}\right) V$.

- Para obtenção do valor da corrente no LED, foi utilizado a tensão obtida na entrada $A_{1}$, pois com ela é possível calcular a corrente que percorre o resistor utilizando a lei de Ohm. Como este resistor encontra-se ligado em série com LED, a corrente do LED é a mesma do resistor. A expressão utilizada para o cálculo foi: $I=\frac{V_{A_{1}}}{120 \Omega}$.

- Finalmente, utilizando uma interface que permite a comunicação do Arduino com o Excel o gráfico da curva corrente $\mathrm{X}$ tensão do laser pode ser obtida no Excel.

\section{A comunicação do Arduíno com o computador}

Queremos transferir dados das medições do Arduíno para o Excel, um editor de planilhas da Microsoft que possui ferramentas de cálculo e construção de gráficos.

\subsection{Procedimentos necessários para transferir dados das medições do Arduino para o Excel}

a) Escrever o programa para o Arduíno (sketch) para transferir os dados das medições para o Excel;

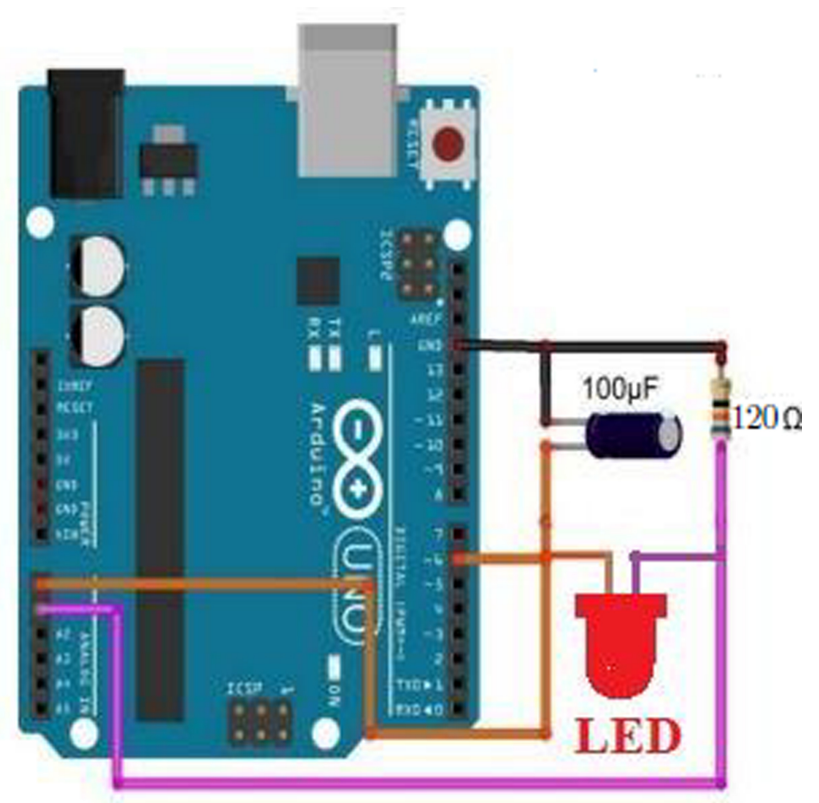

Figura 12: Montagem do circuito eletrônico ligado ao Arduino Uno. 


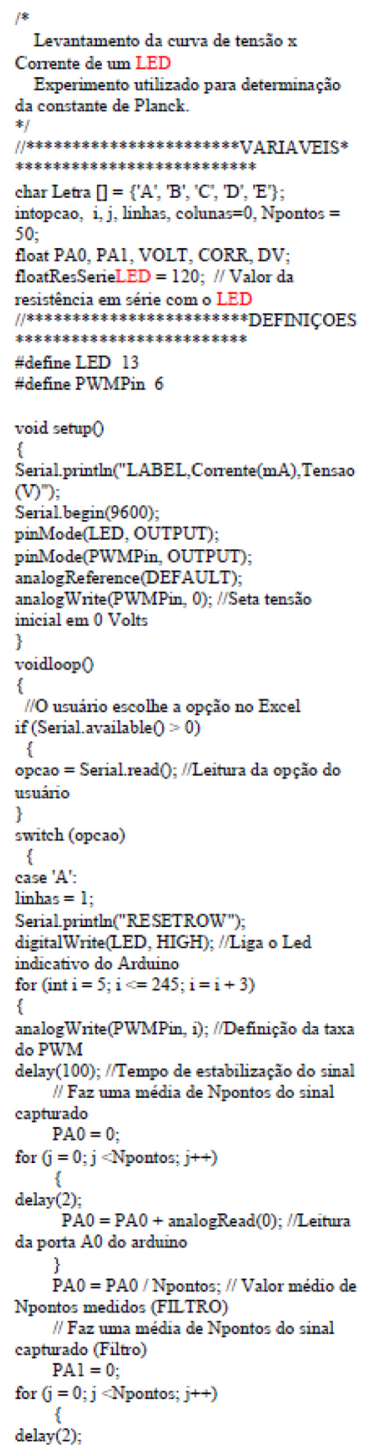

b) Instalar um programa, uma Macro PLX-DAQ, no computador, que vai permitir que o Excel receba os dados do Arduíno.

\subsection{O programa para o Arduíno}

Abaixo é dado o programa (Sketch) para o Arduíno em conformidade com o esquema eletrônico escolhido.

\section{Apresentação dos resultados das medições}

\subsection{A regressão linear}

Para a obtenção da tensão limiar $U_{o}$ da emissão de radiação LED, torna-se necessário aplicar o método dos mínimos quadrados para ajustar a contribuição da parte quase retilínea das medições no gráfico através da regressão linear. O ajuste da curva obtida é dado pela função $\mathrm{y}=\mathrm{ax}+\mathrm{b}$, equação da reta (variação linear). Omitindo-se os cálculos matemáticos, as expressões para o parâmetro $a$ (coeficiente angular) e para o parâmetro $b$ (coeficiente linear) procurados, têm as seguintes formas:

$$
\begin{gathered}
a=\frac{n \sum_{i=1}^{n} x_{i} y_{i}-\sum_{i=1}^{n} x_{i} \sum_{i=1}^{n} y_{i}}{n \sum_{i=1}^{n} x_{i}^{2}-\left(\sum_{i=1}^{n} x_{i}\right)^{2}} \\
b=\frac{1}{n}\left(\sum_{i=1}^{n} y_{i}-a \sum_{i=1}^{n} x_{i}\right)
\end{gathered}
$$

O valor do erro médio quadrático da aproximação examinada também pode ser obtido com o auxílio da teoria dos erros de medição das grandezas físicas 16 . Entretanto, utilizamos as ferramentas de cálculo e construção de gráficos do Excel na elaboração dos resultados. Foram realizadas medições da dependência $\mathrm{I}(\mathrm{mA})$ vs $\mathrm{V}$ (volt) dos dispositivos eletrônicos LEDs e, com o auxílio do software Excel foram obtida as curvas características dos mesmos.

\subsection{Gráficos da curva característica obtida com o Excel}

A solução utilizada para comunicação da plataforma Arduíno com o software Excel foi a ferramenta Parallax Data Acquisition tool (PLX-DAQ).

Esta ferramenta de software é uma add-in gratuita que pode ser agregada ao Microsoft Excel. Com ela, é possível estabelecer uma comunicação serial bidirecional do Computador com um Microcontrolador. O PLX-DAQ recebe e organiza os dados enviados pelo microcontrolador em linhas e colunas dentro de uma planilha Excel, tudo isto em tempo real. Depois de instalado, o PLX-DAQ pode receber várias customizações. Na Figura 13 é apresentada a customização efetuada e utilizada neste trabalho.

Para ativar o PLX-DAQ basta pressionar as teclas Ctrl + Q na planilha Excel. Como pode ser observado no código do programa instalado no Arduíno, somente dois botões foram necessários para este experimento. O botão denominado "Coletar Curva", o qual é responsável por enviar o caractere "A"para o Arduíno.

No Arduíno, uma vez que este caractere é recebido, ele é armazenado na variável intitulada opção. Desta forma, o fluxo de código toma a decisão de coletar os dados de corrente e tensão do LED, enviando-os para a planilha Excel. Esta rotina é parcialmente apresentada na Figura 14 Caso o botão denominado "Zerar Planilha"seja selecionado no PLX-DAQ, o caractere "B"será enviado

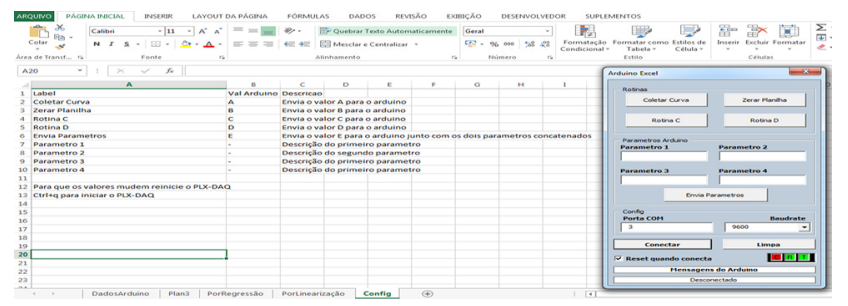

Figura 13: Configuração utilizada no PLX-DAQ. 


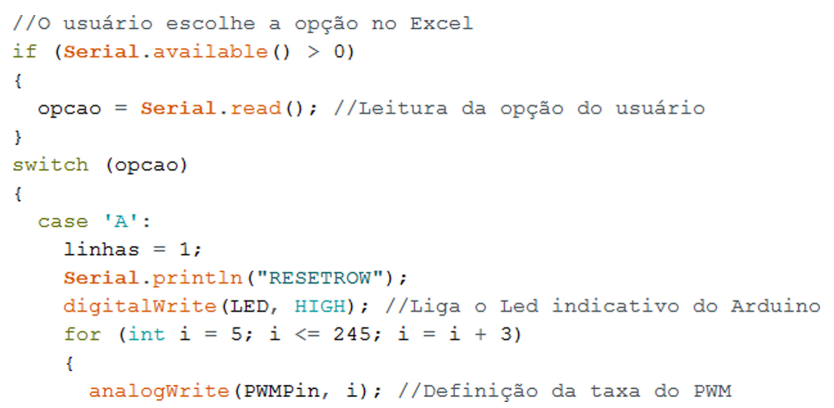

Figura 14: Código para a escolha da captura de dados e envio para a planilha Excel.

para o Arduíno. E, assim, todos os dados da planilha serão apagados.

Um trecho da rotina de código do programa instalado no microcontrolador da plataforma Arduíno que executa esta operação é apresentado na Figura 15

De forma simplificada, o processo de obtenção da curva Corrente x Tensão no LED se dá da seguinte maneira: uma vez que a opção "Coletar Curva"for escolhida, o Arduíno iniciará o processo de aumentar passo a passo o comprimento do pulso PWM, e consequentemente a tensão de alimentação do circuito no qual o LED encontra-se interligado. Os valores analógicos de tensão sobre o LED e resistor são obtidos. Para minimizar ruídos característicos da conversão analógico digital, foi implementado um filtro digital passa baixa utilizando a operação de média. Os dados de corrente e tensão do LED, depois de tratados, são enviados para a planilha Excel (Figura 16).

Os gráficos das curvas características Corrente x Tensão dos LEDs coloridos estudados são então plotados em tempo real. A Figura 17 mostra as curvas características dos LEDs coloridos com as respectivas regressões lineares.

\section{Determinação da constante de Planck}

As determinações da constante de Planck foram obtidas de acordo com as características dos LEDs coloridos estu-

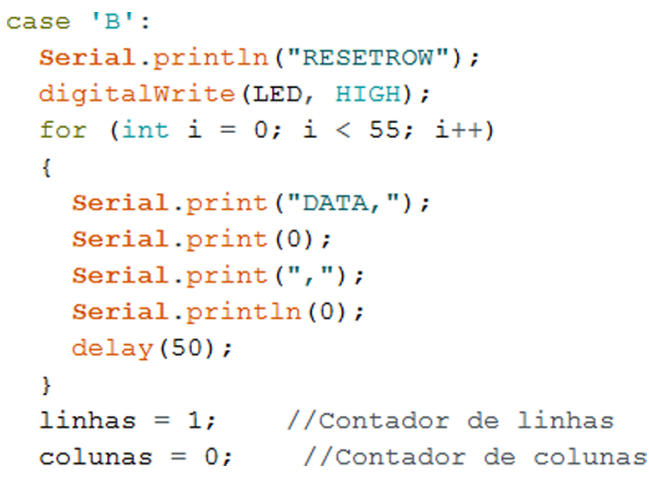

Figura 15: Código para escolha da remoção dos dados da planiIha Excel. dados, aplicando-se a fórmula (7), seção V. Nesse sentido, foram aplicadas as ferramentas de cálculo e construção de gráficos do Excel, com os dados da Tabela 1.

Abaixo, nas Tabelas 2, 3 e 4, são dados os valores determinados da constante de Planck para os LEDs vermelho, verde e azul estudados experimentalmente.

\section{Considerações finais}

Para determinação da constante de Planck foram construídos dois modelos de maquete experimental automatizada (Figura 5 e Figura 8), cuja diferença entre eles consiste no método de observação do espectro de difração da radiação emitida pelo LED numa régua graduada.

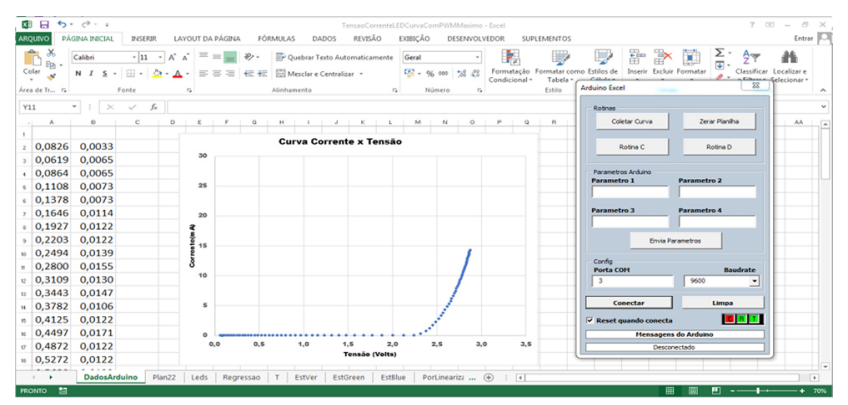

Figura 16: Curva Corrente x Tensão (Planilha do Excel).

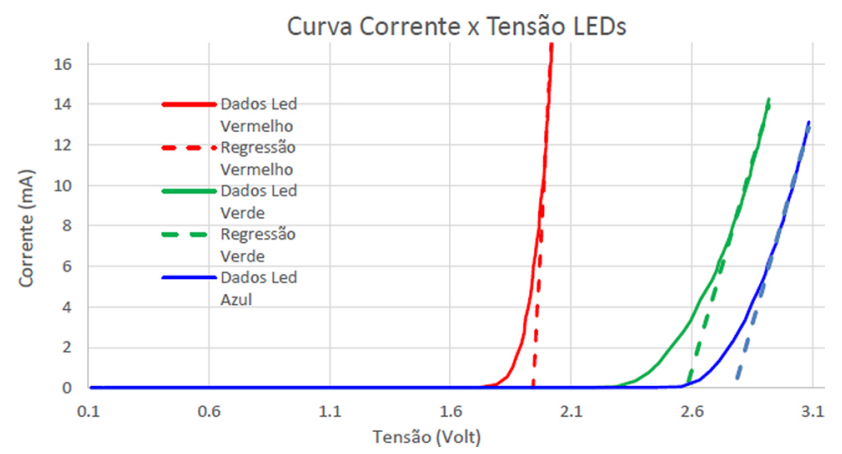

Figura 17: Curvas características de LEDs coloridos com regressões lineares obtidas com o auxílio do Excel.

Tabela 1: Parâmetros para o cálculo da constante de Planck.

\begin{tabular}{lccc}
\hline $\begin{array}{l}\text { Dist. da rede de } \\
\text { difração para régua } \\
\text { graduada }\end{array}$ & $x$ & 171 & $\mathrm{~mm}$ \\
Dist. máx.sec. & & & \\
vermelho & $y_{\text {1vermelho }}$ & 140 & $\mathrm{~mm}$ \\
Dist. máx sec. verde & $y_{\text {1verde }}$ & 98 & $\mathrm{~mm}$ \\
Dist. máx. sec. azul & $y_{1 \text { azul }}$ & 87 & $\mathrm{~mm}$ \\
Angulo teta vermelho & $\theta_{\text {mvermelho }}$ & 0,6889 & $\mathrm{rad}$ \\
Angulo teta verde & $\theta_{\text {mverde }}$ & 0,5229 & $\mathrm{rad}$ \\
Angulo teta azul & $\theta_{\text {mazul }}$ & 0,4730 & $\mathrm{rad}$ \\
Carga do elétron & $e$ & $1,602 \times 10^{-19}$ & $\mathrm{C}$ \\
Período da Rede & $d$ & $1,0 \times 10^{-6}$ & $\mathrm{~m}$ \\
Ordem da difração & $m$ & 1 & \\
Velocidade da Luz & $c$ & $3,0 \times 10^{8}$ & $\mathrm{~m} / \mathrm{s}$ \\
Const.Planck-valor & & & \\
tabela & $h$ & $6,626 \times 10^{-34}$ & $\mathrm{~J} . \mathrm{s}$
\end{tabular}


Um dos modelos (Figura 8 possui uma lente convergente entre o LED e a rede de difração, para colimar a radiação dispersa do LED. A vantagem da utilização do modelo de maquete mostrado na Figura 8 está em que não é necessário escurecer o ambiente para a visão do espectro da luz difratada projetado na régua graduada. O experimento realizado segue a crescente tendência de uso da plataforma Arduíno na automatização dos experimentos de Física Geral por oferecer uma série de vantagens em relação a outros dispositivos devido ao seu ambiente de programação simples e claro, preços baixos e uma infinidade de placas de expansão. Assim, na maquete experimental automatizada, construída para a determinação da constante de Planck, temos sistemas eletrônicos e de software que a controlam atualizados.

A principal atualização no sistema de controle é a interface de comunicação com o computador através do protocolo USB. Ele é baseado no microcontrolador ATMEL328 por meio da plataforma Arduíno. É importante observar que essa plataforma é um projeto de circuito de código aberto, baseado em microcontrolador da indústria Atmel. Uma placa física em código aberto baseada em um circuito de entradas e saídas simples. A mesma já possui conversor analógico/digital. Assim, é possível gerenciar o controle da maquete automatizada usando a interface USB do computador, por meio de um dispositivo de baixo custo. A escolha do LED no experimento para a determinação da constante de Planck é interessante por-

Tabela 2: Determinação da constante de Planck para o LED VERMELHO.

\begin{tabular}{lccc}
\hline Valor da tensão limiar & $U_{o}$ & 1,893 & Volt \\
Coeficiente angular & & & \\
da reta & $a$ & 225,47 & \\
Coeficiente linear da reta & $\mathrm{b}$ & $-426,77$ & \\
Const. de Planck & & & \\
determinada & $h^{\prime}$ & $6,425 \times 10^{-34}$ & J.s \\
Erro Percentual & Erro $\%$ & 2,939 & $\%$ \\
\hline
\end{tabular}

Tabela 3: Determinação da constante de Planck para o LED VERDE.

\begin{tabular}{|c|c|c|c|}
\hline Valor da tensão limiar & $U_{o}$ & 2,529 & Volt \\
\hline Coeficiente angular & & & \\
\hline da reta & $a$ & 40,98 & \\
\hline Coeficiente linear da reta & $\mathrm{b}$ & $-103,63$ & \\
\hline $\begin{array}{l}\text { Const. de Planck } \\
\text { determinada }\end{array}$ & $h^{\prime}$ & $6,744 \times 10^{-34}$ & J.s \\
\hline Erro Percentual & Erro \% & 1,879 & $\%$ \\
\hline
\end{tabular}

Tabela 4: Determinação da constante de Planck para o LED AZUL.

\begin{tabular}{lccc}
\hline Valor da tensão limiar & $U_{o}$ & 2,724 & Volt \\
Coeficiente angular & & & \\
da reta & $a$ & 41,66 & \\
Coeficiente linear da reta & $\mathrm{b}$ & $-113,57$ & \\
Const. de Planck & & & \\
determinada & $h$ & $6,633 \times 10^{-34}$ & J.s \\
Erro Percentual & Erro $\%$ & 0,192 & $\%$ \\
\hline
\end{tabular}

que suas aplicações estão presentes em diversas esferas importantes da vida cotidiana. O mais importante, no entanto, é que o LED é uma ligação vital entre eletrônica e fotônica.

A automatização das medições e da elaboração dos dados dos experimentos de Física tem como objetivo, em primeiro lugar, garantir aos estudantes do Curso de Licenciatura em Física da UESB, modalidade a distância, o acesso aos laboratórios remotos de Física Geral via Internet, mas também a sua utilização pelos estudantes do curso presencial de Física, o que permite aos alunos complementar as aulas teóricas, aprofundar o conhecimento de métodos e técnicas do experimento, estudar aparelhos de pesquisa científica e a familiarização com os mesmos, dominar diferentes métodos de pesquisas experimentais, dominar métodos matemáticos e gráficos dos resultados obtidos e também a avaliação das medições realizadas.

\section{Referências}

[1] H.J. Round, The Electrical World 49, 19 (1907).

[2] The Road to the Transistor, disponível em: https://www.jmargolin.com/history/trans.htm, acessado em 04/05/2017.

[3] O.V. Losev, Philosophical Magazine 5, 39 (1928).

[4] T.H. Lee, The design of CMOS radio-frequency integrated circuits, (Cambridge University Press, Cambridge, 2003).

[5] N. Zheludev, Nature Photonics 1, 4 (2007).

[6] O.V. Losev, Physik. Zeitschr 30, (1929).

[7] H. Júnior e N. Bevacqua, Appl. Phys. Lett. 1, 4 (1962).

[8] H. Wolinsky, U. I.'s Holonyak out to take some of Ediso's luster, (Chicago Sun-Times, Chicago, 2005).

[9] T.P. Perry, IEEE Spectrum 32, 2 (1995).

[10] Brief Biography Holonyak, Craford, Dupuis, disponível em https://www.simplyled.co.uk/blog/a-brief-history-ofthe-led/, acessado em 18/04/2019.

[11] T.S.M. Pearsall, Appl. Phys. Lett. 28, 9 (1976).

[12] The Nobel Prize in Physics 2014, disponível em https://kva.se/nobelphysics2014.

[13] D. Halliday, R. Resnick e K.S. Krane, Física (Ed. LTC, Rio de Janeiro, 2004), v. 4.

[14] S.L. de Moura, F.I da Silva, F.C.M. da Silva e J.A.V. dos Santos, Química Nova na Escola 33, 4 (2011).

[15] Measuring the IV Curve of Semiconductors Whith an Aduino disponível em https://www.instructables.com/id/Measuring-the-IV-Curve-ofSemiconductors-With-an-A/, acessado em 21/09/2018.

[16] I.N. Oliveira, Erros de medição das grandezas físicas e elaboração dos resultados do experimento (Ed. UESB, Vitória da Conquista, 2018)

[17] https://www.youtube.com/watch?v=nJaQxY6yl9Y, acessado em 15/04/2018. 